\title{
TITLE:
}

\section{Low-energy properties of two- dimensional quantum triangular antiferromagnets: Nonperturbative renormalization group approach}

\author{
AUTHOR(S): \\ Fujimoto, S
}

\section{CITATION:}

Fujimoto, S. Low-energy properties of two-dimensional quantum triangular

antiferromagnets: Nonperturbative renormalization group approach. PHYSICAL REVIEW B 2006, 73(18): 184401.

\section{ISSUE DATE:}

2006-05

URL:

http://hdl.handle.net/2433/50023

RIGHT:

Copyright 2006 American Physical Society 
PHYSICAL REVIEW B 73, 184401 (2006)

\title{
Low-energy properties of two-dimensional quantum triangular antiferromagnets: Nonperturbative renormalization group approach
}

\author{
Satoshi Fujimoto \\ Department of Physics, Kyoto University, Kyoto 606-8502, Japan \\ (Received 8 November 2005; revised manuscript received 18 January 2006; published 1 May 2006)
}

\begin{abstract}
We explore low-temperature properties of quantum triangular Heisenberg antiferromagnets in two dimensions in the vicinity of the quantum phase transition at zero temperature. Using the effective field theory described by the $O(3) \times O(2)$ matrix Ginzburg-Landau-Wilson model and the nonperturbative renormalization group method, we clarify how quantum and thermal fluctuations affect long-wavelength behaviors in the parameter region where the systems exhibit a fluctuation-driven first order transition to a long-range ordered state. We show that at finite temperatures the crossover from a quantum $\phi^{6}$ theory to a renormalized twodimensional classical nonlinear sigma model region appears, and in this crossover region, massless fluctuation modes with linear dispersion a la spin waves govern low-energy physics. Our results are partly in good agreement with the recent experimental observations for the two-dimensional triangular Heisenberg spin system, $\mathrm{NiGa}_{2} \mathrm{~S}_{4}$.
\end{abstract}

DOI: 10.1103/PhysRevB.73.184401

PACS number(s): 75.10.Jm, 75.40.-s

\section{INTRODUCTION}

It has been argued for decades that geometrical frustration gives rise to unusual magnetic properties in quantum antiferromagnets, and may bring about an exotic ground state such as a spin liquid which is characterized by the absence of any type of spontaneous symmetry breaking including magnetic long-range order (LRO), and dimerization, etc. ${ }^{1-7}$ The concept of the spin liquid was first proposed by Anderson in connection with the ground state of two-dimensional (2D) Heisenberg antiferromagnets (HAF) on a triangular lattice. ${ }^{7}$ While extensive studies on frustrated magnets have revealed that the triangular HAF may exhibit the magnetic LRO ${ }^{8-18}$ the Anderson's idea is still attracting much interest, and has been tested for its possible realization in other geometrically frustrated systems such as pyrochlore and Kagome HAF, and multiple ring exchange spin models. ${ }^{19-35}$

Recently, Shimizu et al. reported that in an organic Mott insulator with spin $s=\frac{1}{2}$ on a triangular lattice, $\kappa-(\mathrm{ET})_{2} \mathrm{Cu}_{2}(\mathrm{CN})_{2}$, no magnetic LRO is observed down to $32 \mathrm{mK}^{36}$ Their experimental results suggest the possibility of a new kind of a ground state including the spin liquid. Subsequently, Nakatsuji et al. found that in a quasi-2D quantum triangular HAF with $s=1, \mathrm{NiGa}_{2} \mathrm{~S}_{4}$, there is no sign of LRO down to $0.35 \mathrm{~K}$, in spite of the existence of strong anferromagnetic interactions. ${ }^{37}$ In the latter system, the specific heat coefficient $C_{v}$ shows the quadratic-temperature dependence $C_{v} \sim T^{2}$ at sufficiently low-temperatures, and the uniform spin susceptibility is constant in the low-temperature regions, indicating the existence of low-lying massless excitations. The origin of these unexpected low-temperature behaviors has not yet been explained.

On the other hand, from a theoretical point of view, there have been only a few works on low-energy properties in the vicinity of the quantum phase transition at $T=0$ in the $2 \mathrm{D}$ triangular HAF. ${ }^{38-40}$ To understand the above-mentioned experimental observations for quantum triangular HAF precisely, we need to develop a theory which describes quantum critical phenomena in these systems at finite temperatures. In contrast to the quantum case, the 3D classical stacked Heisenberg model, which is equivalent to the $2 \mathrm{D}$ quantum model at $T=0$, has been extensively studied by many authors. ${ }^{8-10,14-18,41-49}$ Even for the classical systems, the elucidation of the nature of the phase transition has not yet been completed. Several theoretical works done by Zumbach, Loison and Schotte, Itakura, and Delamotte and co-workers indicate that the 3D classical triangular HAF show a fluctuation-driven first order phase transition. ${ }^{41-46}$ On the other hand, the loop expansion calculations carried out by Pelissetto et al. and Calabrese et al. support the existence of the continuous phase transition in these systems. ${ }^{47,49}$ Also, most of experiments seem to be in accordance with the continuous transition. ${ }^{50}$ However, the former point of view is quite intriguing, since it implies that the phase transition of the $2 \mathrm{D}$ quantum version of these systems at $T=0$ may be the (quantum) fluctuation-driven first order type. Although the quantum second order phase transition and the related quantum critical phenomena have been comprehensively explored so far, ${ }^{38-40,51-53}$ long-wavelength properties which emerge near the quantum fluctuation-driven first order transition have not been clarified sufficiently. It is well known that in the case of the continuous quantum phase transition, critical phenomena just above the transition point at $T=0$ are described by a renormalized 2D classical theory. In contrast, it is highly nontrivial how quantum and thermal fluctuations which induce a first order transition at zero temperature affect low-energy behaviors (see Fig. 1). In this paper, we would like to address this issue for the $2 \mathrm{D}$ quantum triangular HAF.

Generally, in 2D quantum critical phenomena, the crossover from the 2+1D quantum behaviors to the renormalized 2D classical ones occurs at finite temperatures. For the precise description of the quantum-classical crossover, a promising theoretical approach may be the nonperturbative renormalization group (RG) method. This technique has been applied to classical frustrated magnets by Delamotte et al. 
$\mathrm{T}$

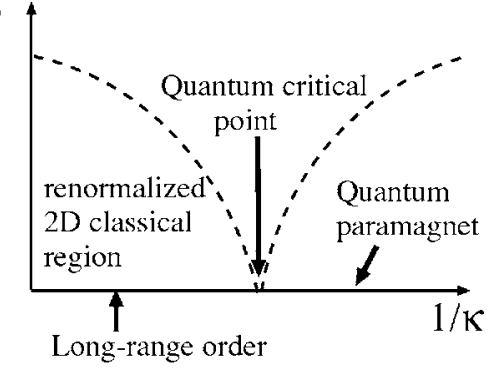

How changed?

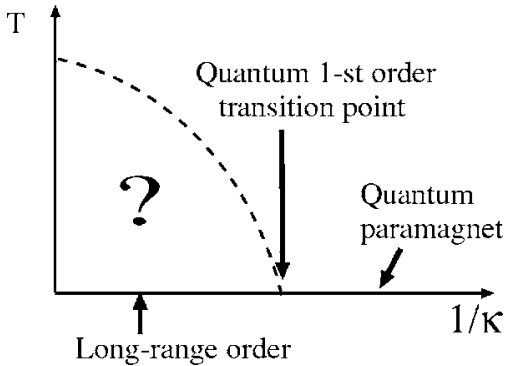

FIG. 1. Phase diagrams of quantum second order phase transition (top) and quantum first order transition (bottom). The vertical and horizontal axes are, respectively, temperature $T$ and a parameter $1 / \kappa$ which controls quantum fluctuations.

yielding fruitful results. ${ }^{46}$ They showed that the nonperturbative RG method successfully reproduces the RG equations for both the 4D Ginzuburg-Landau-Wilson (GLW) model and the 2D nonlinear sigma model, which are the effective field theories for the 4D and 2D classical triangular HAF, respectively, and is expected to capture correct low-energy physics of the 3D triangular HAF. Thus, the nonperturbative RG method may be suitable for the investigation of the dimensional (or quantum-to-classical) crossover phenomena for these systems. We utilize this remarkable merit of the approach to shed light on how quantum and thermal fluctuations control low-energy properties in the vicinity of quantum fluctuation-driven first order transitions.

Our main results are as follows. At finite temperatures, the crossover from a quantum $\phi^{6}$ model to a renormalized $2 \mathrm{D}$ classical system appears, and as $T$ decreases, longwavelength behaviors are almost governed by the $\phi^{6}$ fluctuations which eventually bring about the first order transition. Also, it is found that in this crossover region, thermodynamic properties are effectively determined by gapless excitations $a$ $l a$ spin waves with a linear dispersion. The presence of these low-lying excitations explains partly the experimental observations for $\mathrm{NiGa}_{2} \mathrm{~S}_{4}$; i.e., the quadratic $T$ dependence of the specific heat coefficient, and the finite $T$-independent uniform spin susceptibility at low temperatures. ${ }^{37}$

The organization of this paper is as follows. In Sec. II, the effective field theory and the formulation of the nonperturbative RG method are given. In Sec. III, we present results on the RG flows obtained by solving numerically the RG equations, which demonstrate the existence of strong fluctuations driving the phase transition at $T=0$ to the first order type. In Sec. IV, we show that in the crossover region, quasiGaussian fluctuations dominate low-energy properties, and derive low- $T$ behaviors of the specific heat coefficient and the uniform spin susceptibility. A summary of our results and discussions on the implication for the recent experimental observations for $\mathrm{NiGa}_{2} \mathrm{~S}_{4}$ are given in Sec. $\mathrm{V}$.

\section{EFFECTIVE FIELD THEORY AND NONPERTURBATIVE RENORMALIZATION GROUP METHOD}

\section{A. Quantum $O(3) \times O(2)$ matrix Ginzburg-Landau-Wilson model for frustrated magnets}

Quantum phase transitions in two dimensions which occur at $T=0$ cannot be described by the usual $\phi^{4}$-type Ginzburg-Landau scheme, since the long-range ordered phase exists only at $T=0$, and the order parameter becomes nonzero abruptly just at $T=0$. In the case of $2 \mathrm{D}$ quantum nonfrustrated HAF, the low-energy properties of the quantum phase transition are successfully explained in terms of the $O(3)$ nonlinear sigma model. ${ }^{51}$ In the derivation of the nonlinear sigma model from the HAF, one merely postulates that the correlation length is larger than the lattice constant. Thus the model describes long-wavelength physics of both the ordered phase at $T=0$ and the disordered phase above the transition temperature, that are mainly governed by transverse fluctuations a la spin waves. The nonlinear sigma model is suitable for the description of the 2D quantum phase transition in which the order parameter amplitude is almost frozen, but the strong transverse fluctuations destroy the LRO at finite temperatures.

The extension of the nonlinear sigma model approach to the 2D quantum triangular HAF was achieved by Dombre and Read, and Azaria et al. ${ }^{38,54}$ The order parameter for this case is expressed by a matrix with $O(3) \times O(2)$ symmetry, $\Phi=\left(\vec{\phi}_{1}, \vec{\phi}_{2}\right)$, where $\vec{\phi}_{i}^{\mathrm{t}}=\left(\phi_{1 i}, \phi_{2 i}, \phi_{3 i}\right)$ is an $O(3)$ vector. The $O$ (3) symmetry reflects the spin rotational symmetry. The $O$ (2) symmetry comes from the $E$ representation of the $C_{3 v}$ symmetry of the triangular lattice, which is enlarged to $O(2)$ in the continuum limit. ${ }^{46,55}$ These vectors obey the nonlinear conditions, $\left|\vec{\phi}_{i}\right|^{2}=1, \vec{\phi}_{1} \cdot \vec{\phi}_{2}=0$ corresponding to the $120^{\circ}$ structure ordered state.

Subsequently, however, it was recognized that the $O(3)$ $\times O(2)$ nonlinear sigma model in three dimensions is not a proper low-energy theory for the classical stacked triangular HAF, because the model neglects totally longitudinal (amplitude) fluctuation modes, which importantly induce a fluctuation-driven first order transition. ${ }^{43-46}$ This fact implies that the $2 \mathrm{D}$ quantum version of the $O(3) \times O(2)$ nonlinear sigma model fails to capture the important low-energy physics at $T=0$. To improve the model, one needs to introduce an effective potential which replaces the above nonlinear condition with the relaxed constraints. Then, the correct lowenergy effective theory for $2 \mathrm{D}$ quantum triangular HAF is given by the $O(3) \times O(2)$ matrix GLW model, of which the action is given by

$$
S=S_{\sigma}+S_{4}+S_{6},
$$




$$
\begin{gathered}
S_{\sigma}=\int_{0}^{\beta} d \tau \int d^{2} x\left[\frac{Z \widetilde{\kappa}}{2} \operatorname{tr}\left(\partial_{\mu} \Phi^{\mathrm{t}} \partial_{\mu} \Phi\right)+\frac{\tilde{\omega} \widetilde{\kappa}^{2}}{4} V_{\alpha} V_{\alpha}\right], \\
S_{4}=\int_{0}^{\beta} d \tau \int d^{2} x\left[\frac{\tilde{\lambda} \widetilde{\kappa}^{2}}{4}\left(\frac{\hat{\rho}}{2}-1\right)^{2}+\frac{\tilde{\mu} \widetilde{\kappa}^{2}}{4} \hat{\tau}\right], \\
S_{6}=\int_{0}^{\beta} d \tau \int d^{2} x\left[\frac{\tilde{\lambda}_{6} \widetilde{\kappa}^{3}}{3}\left(\frac{\hat{\rho}}{2}-1\right)^{3}+\tilde{\mu}_{6} \widetilde{\kappa}^{3}\left(\frac{\hat{\rho}}{2}-1\right) \hat{\tau}\right],
\end{gathered}
$$

with $\beta=1 / T$ the inverse temperature, and

$$
\begin{gathered}
V_{a}=\epsilon_{i j}\left(\Phi^{\mathrm{t}} \partial_{a} \Phi\right)_{i j}, \\
\hat{\rho}=\operatorname{tr}\left(\Phi^{\mathrm{t}} \Phi\right), \quad \hat{\tau}=\frac{1}{2} \operatorname{tr}\left(\Phi^{\mathrm{t}} \Phi-\frac{\hat{\rho}}{2}\right)^{2} .
\end{gathered}
$$

Here, $\partial_{\mu}=\left(\frac{1}{c_{1}} \partial_{\tau}, \partial_{x}, \partial_{y}\right), \partial_{a}=\left(\frac{1}{c_{3}} \partial_{\tau}, \partial_{x}, \partial_{y}\right)$. The model (1) describes two massless excitations with the velocity $c_{1}$, and a massless excitation with the velocity,

$$
c_{t}=\sqrt{\frac{Z+\widetilde{\omega} \widetilde{\kappa}}{Z\left(c_{3} / c_{1}\right)^{2}+\widetilde{\omega} \widetilde{\kappa}}} c_{3},
$$

as well as a massive excitation with the mass $\tilde{\lambda} \widetilde{\kappa}$, and two massive excitations with the mass $\tilde{\mu} \widetilde{\kappa}$. The three massless excitations are, respectively, two out-of-plane modes and one in-plane mode of transverse fluctuations a la spin waves. The second part $S_{4}$ is an effective potential which imposes the released nonlinear conditions on $\vec{\phi}_{i}$. In the limit of $\tilde{\lambda} \rightarrow \infty$, $\tilde{\mu} \rightarrow \infty, S_{4}$ recovers the strict nonlinear conditions, and $S_{\sigma}$ $+S_{4}$ becomes equivalent to the action of the $O(3) \times O(2)$ nonlinear sigma model. ${ }^{11,38,54,55}$ The release of the constraint allows the existence of the three massive longitudinal fluctuation modes. The classical version of the model $S_{\sigma}+S_{4}$ has been extensively studied so far. ${ }^{42,44-46}$ Here, we also consider the six-body part $S_{6}$ which is required for the correct description of the fluctuation-driven first order transition. In the expression of $S_{6}$, we neglect terms with derivatives, because the scaling dimensions of these terms imply that they are irrelevant. Although in the following our analysis is applied to nonperturbative regions including the strong-coupling limit, we believe that the omission of the six-body terms with derivatives would not change the essential features of our results. Then, since any polynomials of $\phi_{i}$ which preserve the $O(3) \times O(2)$ symmetry are expressed in terms of $\hat{\rho}$ and $\hat{\tau}^{46}$ six-body terms are generally given by Eq. (4). The effective field theory (1) captures low-energy physics of the 2D quantum triangular HAF in the vicinity of the long-range ordered state with the $120^{\circ}$ structure, which are governed by both quantum and thermal fluctuations. The $120^{\circ}$ structure state is expressed by the configuration of $\Phi$ which minimizes $S$, i.e., $\delta S / \delta \phi_{i}=0$. Transverse and longitudinal spin fluctuations around this configuration which preserve relative angles between spins on a primitive triangle are included in the model (1).

It is noted that although the condition $\delta S / \delta \phi_{i}=0$ leads the finite amplitude of the order parameter, the model is also applicable to the disordered phase at finite temperatures, since in $2 \mathrm{D}$ systems transverse fluctuations for $T>0$ are so strong that the average magnetization vanishes even under this condition, in accordance with the Mermin-WagnerColeman theorem. ${ }^{56}$ This is a key feature of the nonlinear sigma model as an effective field theory for the 2D quantum phase transition at $T=0$. We would like to stress again that any perturbative approaches for the GLW model (1) cannot describe the $2 \mathrm{D}$ quantum phase transition properly because of the reason explained above, and a theoretical framework which can interpolate the nonlinear sigma model and the GLW model is required. The nonperturbative RG method is most suitable for this purpose, as will be explained in the following sections.

\section{B. Nonperturbative renormalization group method for quantum phase transitions}

We apply the nonperturbative RG method to the model (1). This approach was developed by Wetterich, Zumbach, and Delamotte et al. for classical matrix GLW models in connection with frustrated magnets. ${ }^{41,42,46,57-59}$ A remarkable merit of this method is that in contrast to perturbative RG calculations, it is applicable to the whole range of the coupling constants $\lambda$ and $\mu$ including the strong-coupling limit, $\lambda, \mu \rightarrow \infty$. In fact, Delamotte et al. showed that the nonperturbative RG method successfully reproduces the RG equations for both the 4D GLW model in the weak-coupling limit and the $2 \mathrm{D}$ nonlinear sigma model in the strong-coupling limit. ${ }^{46}$ This feature is quite important in the investigation of the quantum phase transition, since our systems may exhibit the quantum-classical crossover at finite temperatures, which is equivalent to the dimensional crossover from the 2D classical region to the $2+1(=3) \mathrm{D}$ quantum-fluctuationdominated region. We exploit this fascinating advantage of the nonperturbative RG method in the following.

It is straightforward to generalize the derivation of the RG equations for the classical system to the $2 \mathrm{D}$ quantum case. ${ }^{60-63}$ We assume that the effective action $\Gamma_{k}[\phi]$ for any values of a scaling parameter $k$ has the same form as Eq. (1) with the renormalized parameters; i.e., $\Gamma[\phi]=S$. This means that the effective action is truncated up to the six-body term. To argue quantum critical behaviors at finite temperatures, we utilize a nonrelativistic renormalization scheme, ${ }^{51,52}$ in which the infrared cutoff is introduced in the momentum space as a scaling parameter $k$. The exact renormalization group equation for the quantum effective action $\Gamma_{k}[\phi]$ is given by

$$
\partial_{t} \Gamma_{k}[\phi]=\frac{1}{2} \sum_{p, p^{\prime}} \widetilde{\partial}_{t} \ln \left[\Gamma_{k}^{(2)}+\frac{T}{(2 \pi)^{2}} R_{k}\left(q^{2}\right) \delta\left(p+p^{\prime}\right)\right],
$$

where $t=-\ln k, p=\left(i \omega_{n}, \vec{q}\right) . \omega_{n}$ is the Matsubara frequency $2 \pi n T$, and $\vec{q}$ is the $2 \mathrm{D}$ momentum. $R_{k}\left(q^{2}\right)$ is the infrared cutoff function in the momentum space. $\widetilde{\partial}_{t}$ acts only on $R_{k}\left(q^{2}\right)$. In the following, we use the theta cutoff function:

$$
R_{k}\left(q^{2}\right)=Z\left(k^{2}-q^{2}\right) \Theta\left(k^{2}-q^{2}\right) .
$$

$\Gamma_{k}^{(2)}$ is the inverse one-particle Green function defined by 


$$
\left\{\Gamma_{k}^{(2)}\left(p, p^{\prime}\right)\right\}_{\alpha \beta}=\left.\frac{\delta^{2} \Gamma_{k}[\phi]}{\delta \phi_{\alpha}(p) \delta \phi_{\beta}\left(p^{\prime}\right)}\right|_{\text {min }} .
$$

Here, the derivative is taken at the configuration which minimizes $\Gamma_{k}[\phi]=S$.

To investigate the renormalization group flow of the effective action (1), we introduce dimensionless renormalized couplings, ${ }^{46}$

$$
\begin{gathered}
\kappa=\frac{Z k^{1-d}}{c_{0}} \widetilde{\kappa}, \quad \lambda=\frac{c_{0} \tilde{\lambda}}{Z^{2} k^{3-d}}, \quad \mu=\frac{c_{0} \tilde{\mu}}{Z^{2} k^{3-d}}, \\
\omega=\frac{c_{0} \tilde{\omega}}{Z^{2} k^{1-d}}, \quad \lambda_{6}=\frac{c_{0}^{2}}{Z^{3}} k^{2 d-4} \tilde{\lambda_{6}}, \quad \mu_{6}=\frac{c_{0}^{2}}{Z^{3}} k^{2 d-4} \tilde{\mu}_{6} .
\end{gathered}
$$

Here $c_{0}$ is an initial value of $c_{1}$. The nonperturbative RG equations for these couplings and the velocities $c_{1}$ and $c_{3}$ are given in the Appendix. In the next sections, we present the results obtained from numerical solutions of the RG equations.

\section{RENORMALIZATION GROUP FLOWS FOR DIMENSIONLESS COUPLINGS AND THE PHASE DIAGRAM}

For the characterization of the quantum phase transition, it is convenient to introduce a dimensionless renormalized temperature $T^{\prime}=T /\left(c_{0} k\right)$ with $c_{0}$ an initial value of $c_{1}$. At finite temperatures, in the scaling limit $k \rightarrow 0$, the renormalized Matsubara frequency $\omega_{n}=2 \pi n T^{\prime}$ for $n \neq 0$ becomes infinity, and thus does not contribute to low-energy properties. Then, in this limit, the system is in the class of the 2D classical model, in which quantum effects are entirely included in the renormalization of parameters. As will be seen below, these renormalized 2D classical behaviors appear only in the sufficiently long-wavelength scale $k \ll k_{c}$, where the critical value of the scaling parameter $k_{c}$ is proportional to $T$.

We solve the RG equations (A1)-(A9) numerically for some particular sets of initial values of parameters by using a Runge-Kutta-Verner method with high precision. We put $k$ $=1$ at the initial stage of the renormalization. Depending on the initial values of the parameters, there are two regions in the scaling limit at $T=0$ as indicated in Fig. 1; i.e., a longrange ordered phase, and a quantum disordered phase (quantum paramagnet). The value of $1 / \kappa$ at the phase boundary depends on the choice of the initial values of the other parameters. The correspondence between the spin $S$ triangular HAF with the nearest-neighbor exchange interaction $J$ and the $O(3) \times O(2)$ nonlinear sigma model implies that the initial values of parameters are set to ${ }^{54}$

$$
\begin{gathered}
c_{1}=\frac{3 \sqrt{3}}{2 \sqrt{2}} J S a, \quad \widetilde{\kappa}=\frac{\sqrt{3}}{4} J S^{2}, \quad Z=1, \\
\widetilde{\omega}=0, \quad \frac{\widetilde{\omega}}{c_{3}^{2}}=-\frac{16}{27 \sqrt{3} J^{3} S^{4} a^{2}},
\end{gathered}
$$

where $a$ is the lattice constant. These parameters are in the region where the LRO exists at $T=0$, and thus we concen-
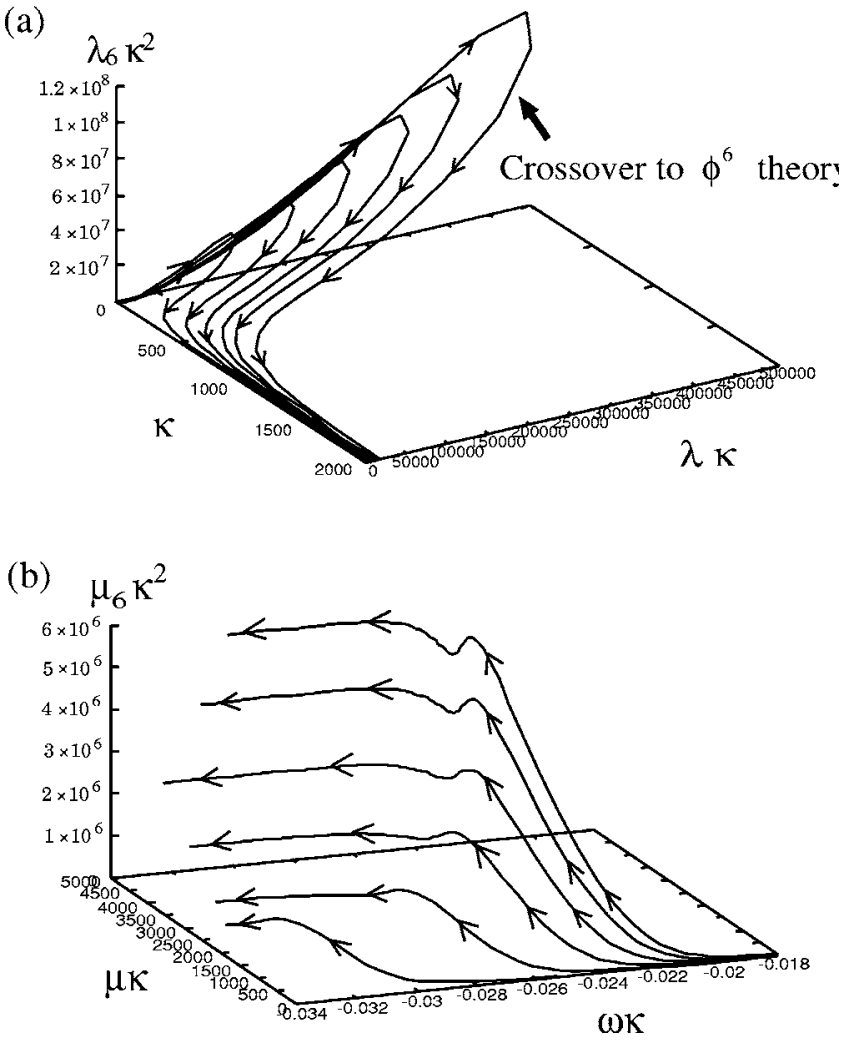

FIG. 2. RG flows of dimensionless couplings. (a) Plots of $\kappa, \lambda \kappa$, and $\lambda_{6} \kappa^{2}$. (b) Plots of $\mu \kappa, \omega \kappa$, and $\mu_{6} \kappa^{2}$. Arrows indicate the directions of the RG flows. The crossover to the $\phi^{6}$ theory appears in the intermediate regions.

trate on this case in the following. As will be seen below, as long as the initial values of parameters are in this region, the qualitative and essential features of the RG flows are not altered by changing the parameters from those given by (13).

In Fig. 2, we show the RG flows obtained at $T=0.001 c_{0}$ for some initial values of parameters. Since the parameter $\kappa$, which is the overall coefficient of the action (1), scales to infinity as $k \rightarrow 0$, we plot the running coupling constants of the four-body and six-body terms, $\lambda \kappa^{2}, \mu \kappa^{2}, \lambda_{6} \kappa^{3}$, and $\mu_{6} \kappa^{3}$, divided by $\kappa$ to specify the low-energy behaviors. The numerical solutions for the RG equations (A1)-(A9) show that the nature of the phase transition and long-wavelength behaviors of the model (1) are different from those predicted for the nonlinear sigma model. ${ }^{38}$ We do not find any nontrivial fixed points which separate the long-range ordered and disordered states, in agreement with the recent studies on the 3D classical stacked triangular HAF. ${ }^{42-46}$ As the scaling parameter $k$ decreases, $\lambda \kappa$ and $\mu \kappa$ scale to large values, implying that at finite temperatures the system is renormalized toward the class of the renormalized 2D classical nonlinear sigma model. However, it should be noted that in the intermediate region, the six-body fluctuations characterized by the parameters $\lambda_{6} \kappa^{2}$ and $\mu_{6} \kappa^{2}$ develop strongly, which may eventually induce a fluctuation-driven first order transition at $T=0$.

To see the crossover behavior toward the $\phi^{6}$ model in the intermediate scale more clearly, we depict the renormalization of the effective potential $S_{4}+S_{6}$ for some values of the 

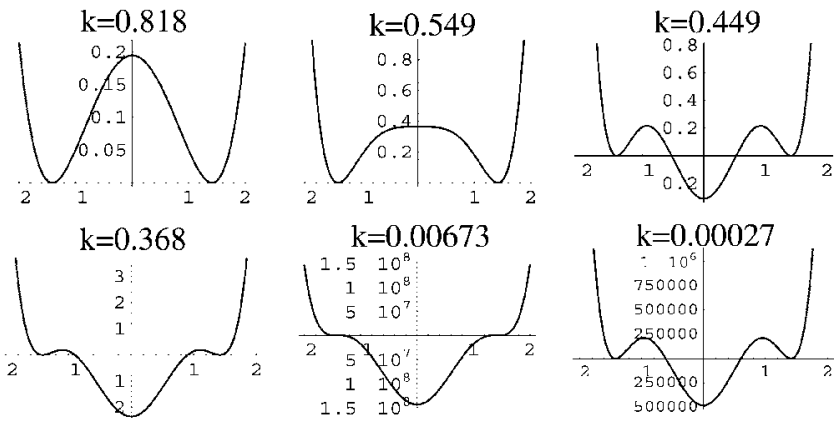

$\mathrm{k}=0.00673$
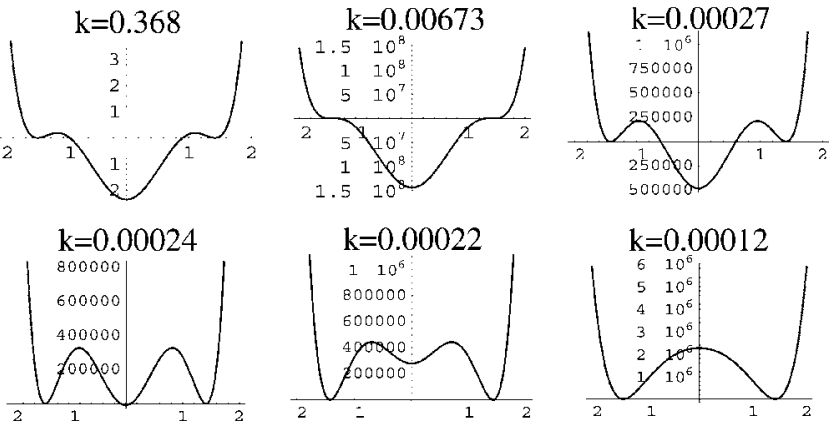

FIG. 3. Effective potentials $S_{4}+S_{6}$ as a function of $\sqrt{\hat{\rho}}$ calculated for a particular set of scaling parameters $k$ under the condition $\hat{\tau}$ $=0$.

scaling parameter $k$ at $T=0.001 c_{0}$ in Fig. 3. Here, for simplicity, we plot the effective potential as a function of $\sqrt{\hat{\rho}}$ under the condition $\hat{\tau}=0$. In the early stage of the renormalization $(k=0.818$ in Fig. 3), the effective potential has two minima at $\sqrt{\hat{\rho}}= \pm \sqrt{2}$, corresponding to the nonlinear condition which expresses the situation that longitudinal fluctuations are suppressed, but strong tranverse fluctuations inhibit the emergence of the LRO. As $k$ decreases, a minimum at $\sqrt{\hat{\rho}}=0$ appears, and the depth of the valley at the origin becomes deeper and deeper, indicating that the fluctuations which may drive the phase transition into the first order type is developing. At $k=0.00673$ in Fig. 3, however, the growth of the minimum at the origin stops and turns to a decline for $k<0.00673$. Eventually, for sufficiently small $k(k$ $=0.00012$ in Fig. 3), the minimum at the origin disappears and the potential valleys at finite $\sqrt{\hat{\rho}}$ become deeper. It is noted that at this final stage the effective potential does not describe a $\phi^{4}$ theory, but corresponds to the nonlinear condition, showing that the system is scaled to the renormalized 2D classical nonlinear sigma model. Here we introduce the scale $k_{c}$ which separates the region in which $\phi^{6}$-type fluctuations strongly develop $\left(k>k_{c}\right)$ and the renormalized 2D classical region $\left(k \ll k_{c}\right)$. It is convenient to define $k_{c}$ as the value of $k$ for which the potential depth at the origin and that at a finite value of $\sqrt{\hat{\rho}}$ coincide. (In Fig. $3, k_{c}=0.00024$.) At $k$ $=k_{c}$, the paramagnetic state and the magnetically ordered state are degenerate. If this situation is realized in the limit of $k \rightarrow 0$, the first order transition to the ordered state occurs. Indeed, this happens at $T=0$. In Fig. 4 , we plot $k_{c}$ calculated for several values of temperatures $T$. The results show that $k_{c}$ is proportional to $T$, and the true phase transition which occurs in the limit $k \rightarrow 0$ realizes only at $T=0$ as a first order transition, which is consistent with the results derived for the 3D stacked classical model. ${ }^{43-46} k_{c} \sim T$ is also the scale at which the quantum-classical crossover occurs. ${ }^{52}$ Then, as the temperature decreases toward $T=0$, the renormalized $2 \mathrm{D}$ classical behaviors appear only for length scales much larger than $1 / k_{c}$, and the low-energy physics at finite temperatures

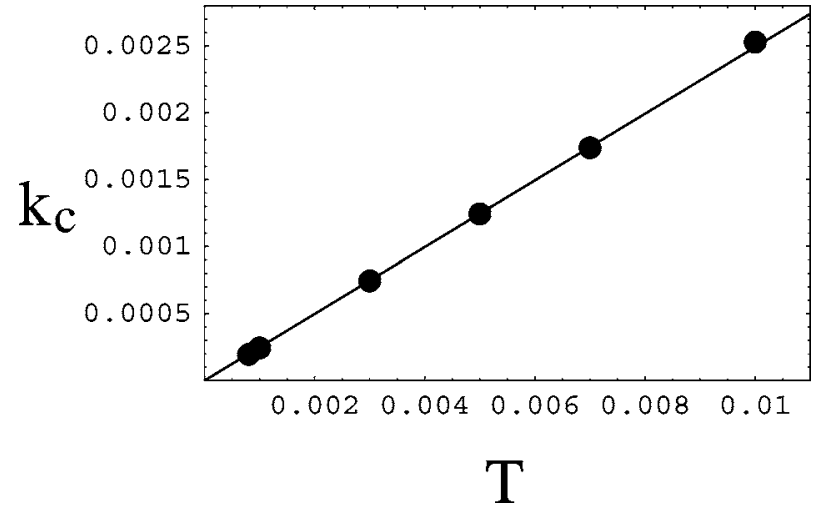

FIG. 4. Plot of the critical scaling parameter $k_{c}$ versus temperature $T$.

are mainly governed by the quantum fluctuations which induce the first order transition. In Fig. 5, we show the schematic phase diagram on the $T-1 / \kappa$ plane suggested from these RG flows for a particular set of the initial values of the other parameters, $\lambda, \mu$, etc. The essential feature of the phase diagram depicted in Fig. 5 does not depend on the choice of these initial values. It is noted that in the long-range ordered state at $T=0$, the effective potential has degenerate minima at the origin and at the nonzero magnetization. This is the unique feature of the quantum first order phase transition for which the transition temperature is $T=0$. The shape of the effective potential implies that there may be a possible coexistence of the ordered and disordered states. The fluctuations which lead the crossover toward the $\phi^{6}$ theory should significantly affect the low-energy properties of the system. We would like to address this issue in the next section.

\section{EMERGENT QUASI-GAUSSIAN LOW-ENERGY BEHAVIORS}

Since the zero-temperature phase transition in our system is the first order type, universal critical behaviors do not

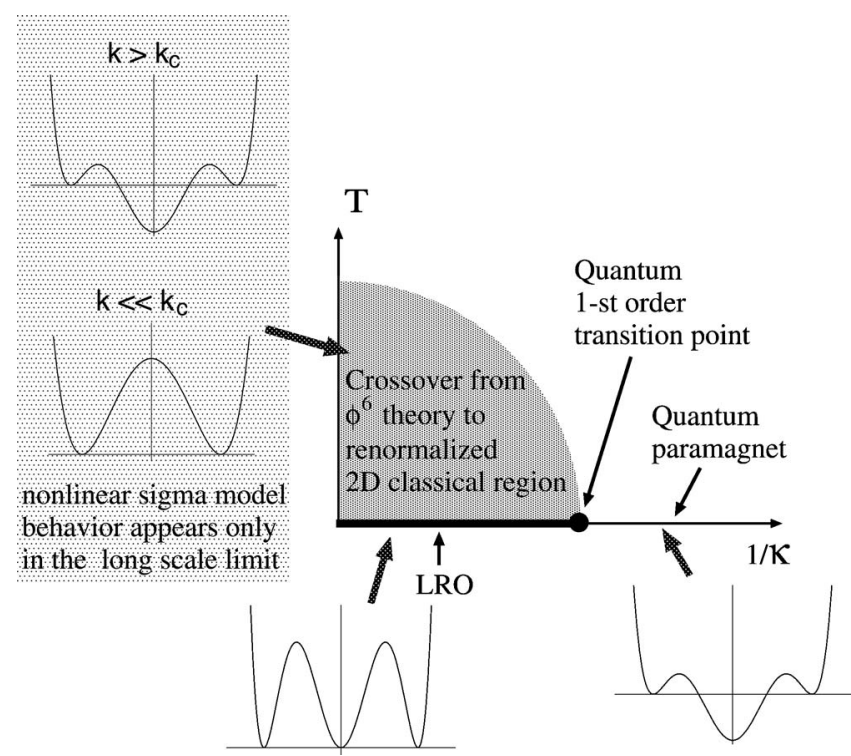

FIG. 5. A schematic phase diagram. The inserted plots are the renormalized potential versus the renormalized field $\sqrt{\hat{\rho}}$. 
(a)

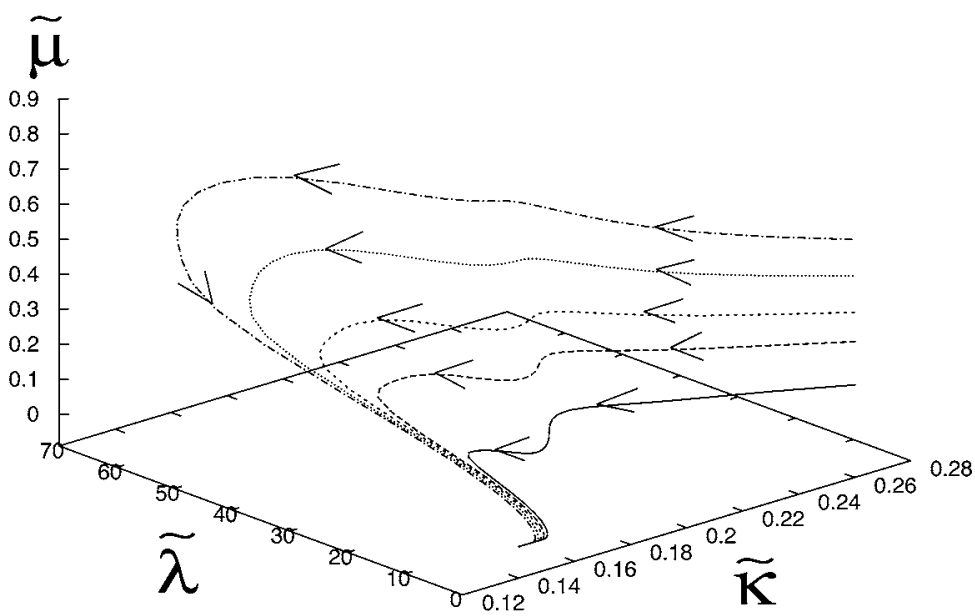

(b)

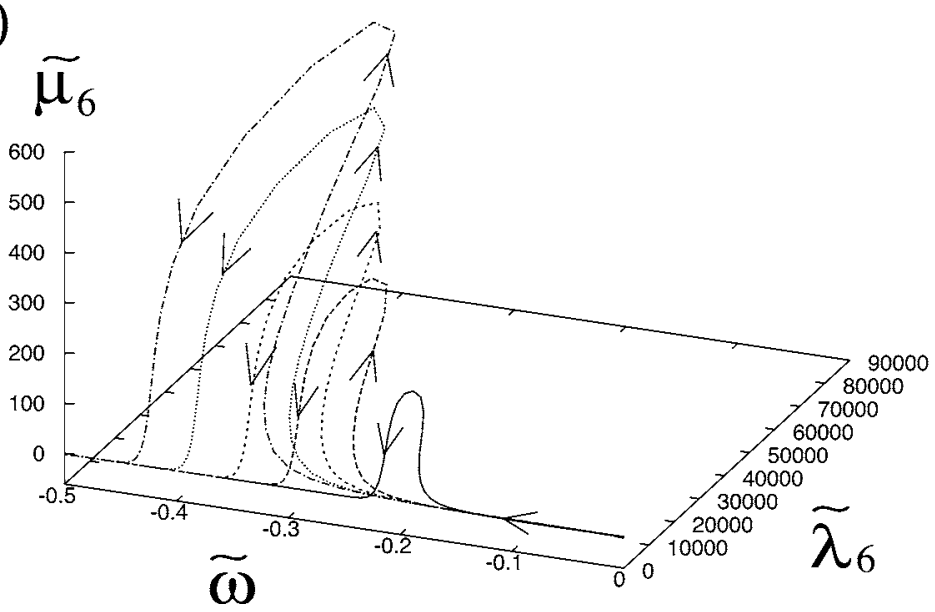

FIG. 6. RG flows of dimensionful couplings for some particular sets of the initial values of parameters. (a) Plots of $\widetilde{\kappa}, \tilde{\lambda}$, and $\tilde{\mu}$. (b) Plots of $\tilde{\omega}, \tilde{\lambda}_{6}$, and $\tilde{\mu}_{6}$. The initial values of parameters are $\tilde{\kappa}(t=0)=0.3, \quad \tilde{\lambda}(0)=0.5, \quad \tilde{\mu}(0)=0.1, \quad \widetilde{\omega}(0)$ $=-0.0001, \quad \widetilde{\omega}(0) / c_{3}^{2}(0)=-0.5, \quad c_{1}(0)=1.0 \quad($ solid line); $\tilde{\kappa}(0)=0.3, \quad \tilde{\lambda}(0)=0.3, \quad \tilde{\mu}(0)=0.22, \quad \widetilde{\omega}(0)$ $=-0.0001, \widetilde{\omega}(0) / c_{3}^{2}(0)=-0.5, c_{1}(0)=1.0$ (broken line); $\quad \tilde{\kappa}(0)=0.3, \quad \tilde{\lambda}(0)=0.3, \quad \tilde{\mu}(0)=0.3, \quad \widetilde{\omega}(0)$ $=-0.0001, \quad \widetilde{\omega}(0) / c_{3}^{2}(0)=-0.5, \quad c_{1}(0)=1.0 \quad($ thin broken line); $\widetilde{\kappa}(0)=0.3, \tilde{\lambda}(0)=0.3, \tilde{\mu}(0)=0.4$, $\widetilde{\omega}(0)=-0.0001, \quad \widetilde{\omega}(0) / c_{3}^{2}(0)=-0.5, \quad c_{1}(0)=1.0$ (dotted line); $\tilde{\kappa}(0)=0.3, \tilde{\lambda}(0)=0.3, \tilde{\mu}(0)=0.5$, $\widetilde{\omega}(0)=-0.0001, \quad \widetilde{\omega}(0) / c_{3}^{2}(0)=-0.5, \quad c_{1}(0)=1.0$ (dotted-and-broken line), and the initial values of both $\lambda_{6}$ and $\mu_{6}$ are equal to 0 in all calculations. exist. However, it is still possible to relate low-energy properties of the model (1) which appeared in a certain parameter region to experimentally observable quantities which are governed by quantum and thermal fluctuations. For this purpose, we investigate the RG flows of dimensionful couplings $\tilde{\kappa}, \tilde{\lambda}, \tilde{\mu}, \tilde{\omega}, \tilde{\lambda}_{6}$, and $\tilde{\mu}_{6}$ for some particular sets of initial values of the parameters, from which physical quantities can be calculated. Surprisingly, as we will see below, longwavelength qualitative behaviors of these running couplings seem to be almost universal to some extent at least in the region where the long-range order realizes at $T=0$, and furthermore, the effective action $\Gamma_{k}[\phi]$ is renormalized to a system in which Gaussian fluctuations dominate low-energy properties.

In Figs. 6 and 7 we show the RG flows for the dimensionful couplings. It is noted that $\tilde{\lambda}, \tilde{\mu}, \tilde{\lambda}_{6}$, and $\tilde{\mu}_{6}$ scale to zero, and $\widetilde{\kappa}$ and $\widetilde{\omega}$ scale to finite nonuniversal constants. Besides, $\widetilde{\kappa} \gg \widetilde{\kappa}^{2} \widetilde{\omega}$ for $k \rightarrow 0$, as long as the initial value of $\widetilde{\kappa}^{2} \widetilde{\omega}$ is much smaller than that of $\widetilde{\kappa}$, which is a proper assumption for our system since the $\widetilde{\omega}$ term [the second term of Eq. (2)] is generated in the process of the renormalization. We would like to stress that these characteristic behaviors are rather universally found for any initial values of the parameters in the region mentioned above. These observations imply that in the scaling limit $k \rightarrow 0$, the effective action is renormalized to a Gaussian-like model, which is given by the first term of Eq. (2) without the nonlinear conditions:

$$
S_{\mathrm{G}}=\int d^{2} x \int d \tau \frac{Z \widetilde{\kappa}}{2} \sum_{i=1}^{2} \partial_{\mu} \vec{\phi}_{i} \cdot \partial_{\mu} \vec{\phi}_{i} .
$$

In the region where the Gaussian-like fluctuation, i.e., the free boson with a linear dispersion, dominates, the specific heat coefficient is easily calculated as,

$$
C_{v}=(3 \sqrt{3} / \pi) \zeta(3)\left(T / c_{1}\right)^{2} .
$$

The spin susceptibility is also obtained from the Gaussian action. For this purpose, we introduce an external in-plane magnetic field $\vec{h}$, which couples with the uniform component of spin fluctuations in the form

$$
-\frac{Z \widetilde{\kappa}}{c_{1}^{2}} \vec{h} \cdot\left(\vec{\phi}_{1} \times \partial_{t} \vec{\phi}_{1}+\vec{\phi}_{2} \times \partial_{t} \vec{\phi}_{2}\right) .
$$

Then, in the quasi-Gaussian region, the spin susceptibility in the limit $T \rightarrow 0$ is a nonzero constant given by $\chi=-\partial^{2} F / \partial h^{2}$ $=k_{0} /\left(12 \pi c_{1}\right)$ with $k_{0}$ an ultraviolet momentum cutoff. The 
(a)

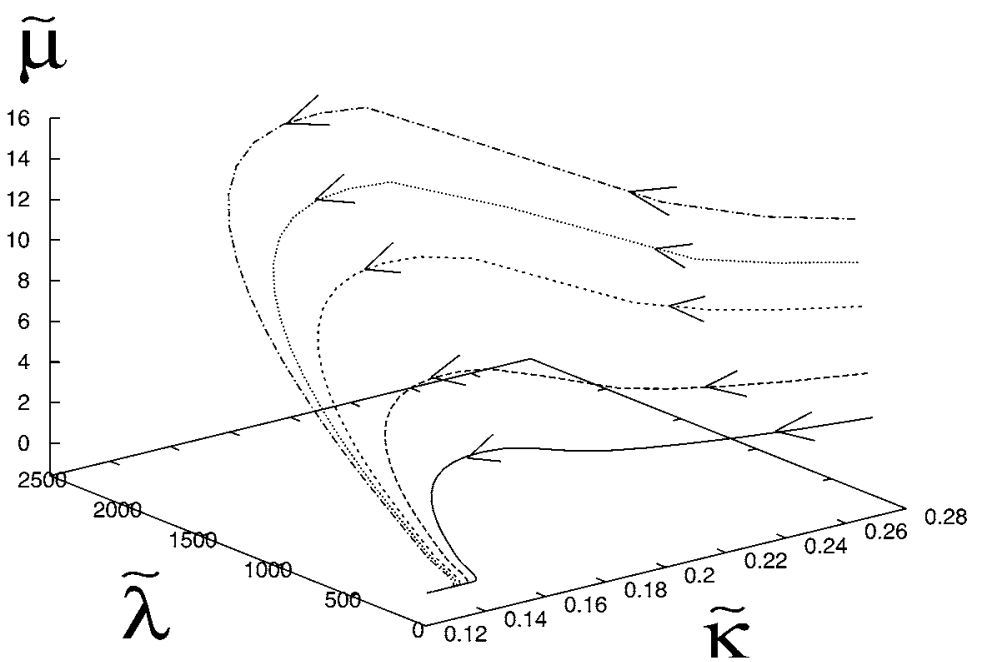

(b)

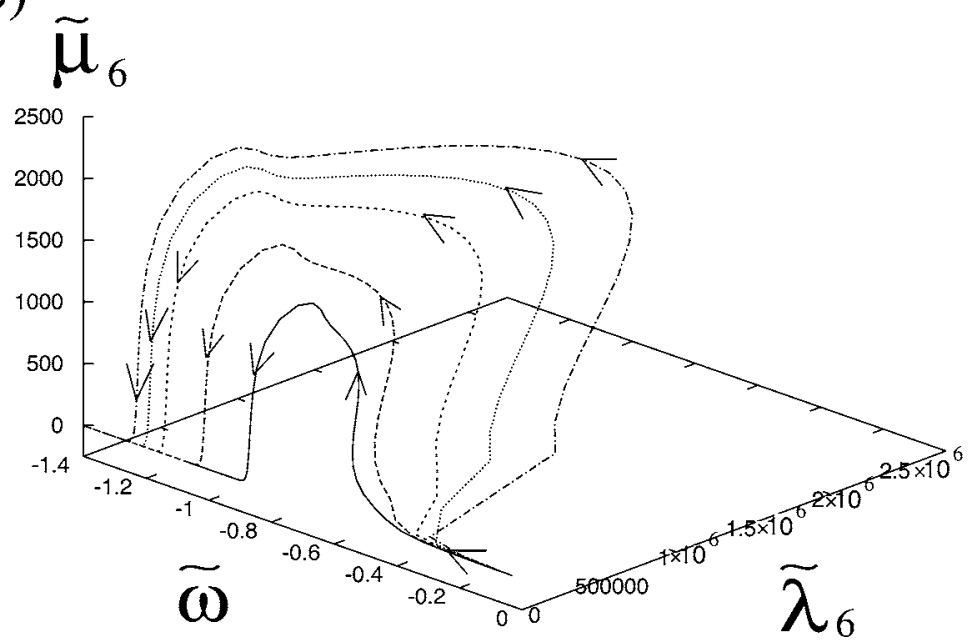

FIG. 7. RG flows of dimensionful couplings. The initial values of parameters are $\widetilde{\kappa}(t=0)$ $=0.3, \quad \tilde{\lambda}(0)=3.0, \quad \tilde{\mu}(0)=3.0, \quad \widetilde{\omega}(0)=-0.0001$, $\widetilde{\omega}(0) / c_{3}^{2}(0)=-0.5, c_{1}(0)=1.0, \quad($ solid line); $\widetilde{\kappa}(0)$ $=0.3, \quad \tilde{\lambda}(0)=5.0, \quad \tilde{\mu}(0)=5.0, \quad \tilde{\omega}(0)=-0.0001$, $\widetilde{\omega}(0) / c_{3}^{2}(0)=-0.5, c_{1}(0)=1.0$ (broken line); $\widetilde{\kappa}(0)$ $=0.3, \quad \tilde{\lambda}(0)=8.0, \quad \tilde{\mu}(0)=8.0, \quad \tilde{\omega}(0)=-0.0001$, $\widetilde{\omega}(0) / c_{3}^{2}(0)=-0.5, c_{1}(0)=1.0$ (thin broken line); $\widetilde{\kappa}(0)=0.3, \quad \tilde{\lambda}(0)=10.0, \quad \tilde{\mu}(0)=10.0, \quad \widetilde{\omega}(0)=$ $-0.0001, \quad \widetilde{\omega}(0) / c_{3}^{2}(0)=-0.5, \quad c_{1}(0)=1.0 \quad($ dotted line); $\tilde{\kappa}(0)=0.3, \tilde{\lambda}(0)=12.0, \tilde{\mu}(0)=12.0, \tilde{\omega}(0)=$ $-0.0001, \widetilde{\omega}(0) / c_{3}^{2}(0)=-0.5, \quad c_{1}(0)=1.0$ (dottedand-broken line), and the initial values of both $\lambda_{6}$ and $\mu_{6}$ are equal to 0 in all calculations.
quasi-Gaussian modes behave like spin waves with a linear dispersion, despite the absence of spontaneous symmetry breaking at finite temperatures. These results seem to explain partly the recent experimental observations for the quasi-2D triangular $\mathrm{HAF} \mathrm{NiGa}_{2} \mathrm{~S}_{4}$ at low temperatures. The spinliquid-like behaviors found in this material at finite temperatures may be attributed to the existence of these fluctuation modes.

\section{SUMMARY AND DISCUSSION}

We have investigated low-energy properties of the $2 \mathrm{D}$ quantum triangular $\mathrm{HAF}$ using the nonperturbative $\mathrm{RG}$ method and the mapping to the $O(3) \times O(2)$ matrix GLW model. Our findings are as follows.

(1) At finite temperatures, at the length scale shorter than $1 / k_{c}$ the $\phi^{6}$ model which describes the fluctuations driving the transition to the first order type dominates the lowtemperature behaviors, while at the scale larger than $1 / k_{c}$ the renormalized $2 \mathrm{D}$ classical region appears. As the temperature is lowered, the crossover scaling parameter $k_{c}$ decreases as $\propto T$, and eventually at $T=0$, the first order phase transition to the $120^{\circ}$ structure occurs.
(2) In the crossover region at finite temperatures, the longwavelength properties are governed by quasi-Gaussian fluctuations a la spin waves with a linear dispersion, which give a quadratic $T$ dependence of the specific heat coefficient and a finite and $T$-independent nonzero value of the uniform spin susceptibility at low temperatures.

Here we would like to discuss the relation between our results and the recent experimental observations for the quasi-2D quantum Heisenberg antiferromagnet on a triangular lattice, $\mathrm{NiGa}_{2} \mathrm{~S}_{4}$, which show no sign of LRO down to $37 \mathrm{mK}$, implying the possible realization of a spin liquid. ${ }^{37}$ At low temperatures, the system exhibits some remarkable properties; (i) The specific heat coefficient shows the quadratic temperature dependence $C_{v} \propto T^{2}$, indicating the existence of gapless excitation modes. (ii) The uniform spin susceptibility for $T \rightarrow 0$ is a nonzero constant, suggesting that magnetic excitations are gapless. (iii) In contrast to the observations (i) and (ii), the magnetic correlation length measured by the neutron scattering is rather short; i.e., $\xi$ $\sim 2.5 \mathrm{~nm}$, leading the authors of Ref. 37 to the conclusion that there may exist gapless nonmagnetic modes. Our results for the specific heat coefficient and the spin susceptibility obtained in Sec. IV seem to be in agreement with the obser- 
vations (i) and (ii), provided that the origin of the gapless excitations may be attributed to magnetic ones, and that in this system the coupling between the triangular layers is so weak that thermal fluctuations suppress the true LRO at experimentally accessible low temperatures. However, the observation (iii) implies that the magnetic excitations may not propagate coherently and have an excitation gap, and is not consistent with our RG analysis which shows the existence of an exponentially long correlation length. Then, how to reconcile our results with the observation (iii)? To explain this point, we would like to note that in our field-theoretical model, chirality domains, which inevitably exist in real triangular HAF and suppress the development of the magnetic correlation length, are not included. Also, as was pointed out by Kawamura and Miyashita, ${ }^{8} Z_{2}$ vortex, which is not taken into account explicitly in the field-theoretical model, may play a crucial role at finite temperatures, disturbing the growth of $\xi^{64,65} \mathrm{It}$ is expected that the velocities of the magnetic excitations, $c_{1,3}$, are strongly renormalized by these topological defects, and reduced to small values. Then, as long as $\xi>c / T$, the magnetic excitations behave like gapless modes, and our results may be applicable. Indeed, the experimental observation (ii) intimates the existence of gapless magnetic excitations. Also, to interpret the experimental observations (ii) and (iii) in a consistent way, one might need to consider effects of randomness such as impurities, which are beyond the scope of this paper, but may play a crucial role in connection with the topological defects inherent in triangular HAF. To confirm this speculation, we need further studies. We would like to address this issue in the near future.

\section{ACKNOWLEDGMENTS}

The author is indebted to B. Delamotte, H. Kawamura, S. Nakatsuji, and H. Tsunetsugu for useful discussions. The numerical computations were partly performed at the Yukawa Institute Computer Facility, Kyoto University. This work was supported by a Grant-in-Aid from the Ministry of Education, Science, Sports, and Culture, Japan.

\section{APPENDIX A: RENORMALIZATION GROUP EQUATIONS FOR THE QUANTUM $O(3) \times O(2)$ GINZBURG- LANDAU-WILSON MODEL}

In this appendix, we present the RG equations for the dimensionless renormalized couplings (11) and (12) for the effective action (1) as functions of the scaling parameter $t$ $=-\ln k$. Here we consider the general case of the spatial dimension $d$. Differentiating Eq. (8) with respect to $\phi_{i}$, as was done in Ref. 46, we end up with the RG equations truncated up to the six-body terms,

$$
\begin{aligned}
\partial_{t} \kappa=- & (d-1+\eta) \kappa-\frac{3}{2} l_{1}(\kappa \lambda)-l_{1}(\kappa \mu)-\frac{r_{10}}{2}-(N-2) l_{1}(0) \\
- & \frac{\omega}{\lambda} r_{11}-2 \frac{\mu}{\lambda} l_{1}(\kappa \mu)-\frac{4 \kappa}{\lambda}\left[\lambda_{6} l_{1}(\kappa \lambda)+2 \mu_{6} l_{1}(\kappa \mu)\right], \\
\partial_{t} \lambda= & (d-3+2 \eta) \lambda-\frac{\lambda^{2}}{4}\left[9 l_{2}(\kappa \lambda)+2 l_{2}(\kappa \mu)+r_{20}+2(N\right. \\
& \left.-2) l_{2}(0)\right]-2 \lambda \mu l_{2}(\kappa \mu)-\lambda \omega r_{21}-\omega^{2} r_{22}-2 \mu^{2} l_{2}(\kappa \mu) \\
& +4 \lambda_{6} l_{1}(\kappa \lambda)+68 \mu_{6} l_{1}(\kappa \mu)-4 \frac{\lambda_{6}}{\lambda}\left[\omega r_{11}+2 \mu l_{1}(\kappa \mu)\right] \\
& -16 \frac{\kappa}{\lambda}\left[\lambda_{6}^{2} l_{1}(\kappa \lambda)+2 \lambda_{6} \mu_{6} l_{1}(\kappa \mu)\right]-16 \lambda_{6}^{2} \kappa^{2} l_{2}(\kappa \lambda) \\
& -32 \mu_{6}^{2} \kappa^{2} l_{2}(\kappa \mu)-12 \kappa \lambda \lambda_{6} l_{2}(\kappa \lambda)-8 \kappa(\lambda \\
+ & 2 \mu) \mu_{6} l_{2}(\kappa \mu), \\
\partial_{t} \mu= & (d-3+2 \eta) \mu-3 \mu \lambda u_{11}(\kappa \lambda, \kappa \mu)-\mu \omega r_{111}(\kappa \mu) \\
& -\frac{\mu^{2}}{2}\left[3 u_{11}(\kappa \lambda, \kappa \mu)+(N-2) l_{2}(0)-r_{110}(\kappa \mu)\right] \\
\eta= & -\frac{\omega}{2} l_{1}(0)-\frac{\kappa \lambda}{2} l_{120}(\kappa \lambda)+\kappa \omega^{2}\left[2 h_{111}(0)+h_{110}(0)\right. \\
& \left.+(1+\kappa \omega) h_{222}(0)+2 r_{111}(0)\right]-\kappa \mu^{2} l_{120}(\kappa \mu), \\
& +4 \mu_{6}\left[l_{1}(\kappa \lambda)+l_{1}(\kappa \mu)-\frac{\omega}{\lambda} r_{11}-\frac{2 \mu}{\lambda} l_{1}(\kappa \mu)\right] \\
& -\frac{16 \kappa}{\lambda}\left[\lambda_{6} \mu_{6}+2 \mu_{6}^{2} l_{1}(\kappa \mu)\right]-32 \kappa^{2} \mu_{6}^{2} u_{11}(\kappa \lambda, \kappa \mu) \\
& -8 \kappa \mu_{6}(\lambda+2 \mu) u_{11}(\kappa \lambda, \kappa \mu), \\
& (\mathrm{A} 3) \\
&
\end{aligned}
$$

$$
\begin{aligned}
\partial_{t} c_{1}= & \frac{c_{1} \omega}{4}\left[1-\frac{c_{1}^{2}}{c_{3}^{2}}\right] l_{1}(0)+\frac{c_{1} \kappa \lambda^{2}}{4}\left[-3 l_{220}(\kappa \lambda)+2 l_{210}(\kappa \lambda)+4 l_{300}(\kappa \lambda)-4 l_{310}(\kappa \lambda)\right]+\frac{c_{1} \kappa \mu^{2}}{4}\left[-3 l_{220}(\kappa \mu)+2 l_{210}(\kappa \mu)\right. \\
& \left.+4 l_{300}(\kappa \mu)-4 l_{310}(\kappa \mu)\right]+\frac{c_{1} \kappa \omega^{2}}{4}\left[4 \frac{c_{1}^{2}}{c_{3}^{2}} r_{111}(0)-r_{121}(0)+4 r_{122}(0)-4 r_{122}(0)-4 r_{132}(0)+4 w_{132}(0)-4 h_{111}(0)-2 h_{111}(0)\right. \\
& \left.-2(1+\kappa \omega) h_{222}(0)-2 r_{111}(0)\right]
\end{aligned}
$$




$$
\begin{aligned}
& \partial_{t} \omega=(d-1+2 \eta) \omega+\frac{\omega}{2 \kappa}\left[l_{1}(\kappa \lambda)-l_{1}(0)\right]-\frac{\lambda^{2}}{2}\left[l_{120}(\kappa \lambda)+2(1+\kappa \omega) h_{220}(\kappa \lambda)\right]+\omega^{2}\left[2 h_{111}(0)+h_{110}(0)+(1+\kappa \omega) h_{222}(0)\right. \\
& \left.+r_{11 \tilde{1}}(0)-6 h_{121}(\kappa \lambda)-(1+\kappa \omega) h_{222}(\kappa \lambda)-r_{111}(\kappa \lambda)-\frac{8}{d} r_{111}(\kappa \lambda)+\frac{8}{d+2} h_{030}(\mu \kappa)+\frac{8}{d+2}(N-2) h_{030}(0)\right]-\mu^{2} l_{120}(\kappa \mu) \\
& +\lambda \omega\left[6 h_{120}(\kappa \lambda)+(1+\kappa \omega) h_{221}(\kappa \lambda)+r_{110}(\kappa \lambda)\right] \\
& \partial_{t} c_{3}=\frac{c_{3} \lambda^{2}}{4 \omega}\left[l_{120}(\kappa \lambda)+2(1+\kappa \omega) h_{220}(\kappa \lambda)-\frac{c_{3}^{2}}{c_{1}^{2}}\left\{-l_{120}(\kappa \lambda)-3 l_{220}(\kappa \lambda)+2 l_{210}(\kappa \lambda)+4 l_{300}(\kappa \lambda)-4 l_{310}(\kappa \lambda)-3 w_{220}(\kappa \lambda)\right.\right. \\
& \left.\left.-10 w_{130}(\kappa \lambda)+4(1+\kappa \lambda) w_{230}(\kappa \lambda)+12(1+\kappa \lambda) w_{140}(\kappa \lambda)\right\}\right]+\frac{c_{3} \omega}{2}\left[2 h_{111}(0)+h_{110}(0)+(1+\kappa \omega) h_{222}(0)+r_{111}(0)\right. \\
& -6 h_{121}(\kappa \lambda)-(1+\kappa \omega) h_{222}(\kappa \lambda)-r_{111}(\kappa \lambda)-\frac{8}{d} r_{11} \tilde{1}(\kappa \lambda)+\frac{8}{d+2} h_{030}(\mu \kappa)+\frac{8}{d+2}(N-2) h_{030}(0)-\frac{1}{2} \frac{c_{3}^{2}}{c_{1}^{2}}\left\{4 \frac{c_{1}^{2}}{c_{3}^{2}} r_{111}(0)\right. \\
& -r_{121}(0)+4 r_{12 \tilde{2}}(0)-4 r_{122}(0)-4 r_{132}(0)+4 w_{132}(0)+6 r_{111}(\kappa \lambda)+8 w_{121}(\kappa \lambda)-16 r_{111}(\kappa \lambda)-16(1+\kappa \lambda) r_{121}(\kappa \lambda) \\
& \left.\left.+16 h_{031}(\mu \kappa)+16(N-2) h_{031}(0)\right\}\right]+\frac{c_{3} \mu^{2}}{2 \omega}\left[-l_{120}(\kappa \lambda)-\frac{c_{3}^{2}}{c_{1}^{2}}\left[-l_{120}(\kappa \lambda)-3 l_{220}(\kappa \lambda)+2 l_{210}(\kappa \lambda)+4 l_{300}(\kappa \lambda)-4 l_{310}(\kappa \lambda)\right]\right] \\
& -\frac{c_{3} \lambda}{2}\left[-6 h_{120}(\kappa \lambda)-(1+\kappa \omega) h_{221}(\kappa \lambda)-r 110(\kappa \lambda)-\frac{c_{3}^{2}}{c_{1}^{2}}\left\{-3 r_{121}(\kappa \lambda)+4 r_{121}(\kappa \lambda)-4 w_{131}(\kappa \lambda)+4 r_{131}(\kappa \lambda)\right\}\right] \text {, } \\
& \partial_{t} \lambda_{6}=(2 d-4+3 \eta) \lambda_{6}+16 \kappa^{2} \lambda_{6}^{3} l_{3}(\kappa \lambda)-30 \kappa \lambda_{6}^{2} l_{2}(\kappa \lambda)-12 \kappa \mu_{6} \lambda_{6} l_{2}(\kappa \mu)-3 \mu \lambda_{6} l_{2}(\kappa \mu)-\frac{3 \omega \lambda_{6}}{2} r_{21}+32 \kappa^{2} \mu_{6}^{3} l_{3}(\kappa \mu)+\frac{\mu^{3}}{2 \kappa} l_{3}(\kappa \mu) \\
& -48 \kappa \mu_{6}^{2} l_{2}(\kappa \mu)+6 \mu_{6} \mu^{2} l_{3}(\kappa \mu)-\frac{39 \mu_{6}}{2 \kappa} l_{1}(\kappa \mu)+24 \kappa \mu_{6}^{2} \mu l_{3}(\kappa \mu)-12 \mu_{6} \mu l_{2}(\kappa \mu)+\frac{\lambda^{3}}{32 \kappa}\left[2(N-2) l_{3}(0)+27 l_{3}(\kappa \lambda)\right. \\
& \left.+2 l_{3}(\kappa \mu)+r_{30}\right]+\frac{27 \lambda^{2} \lambda_{6}}{4} l_{3}(\kappa \lambda)+\frac{3 \mu_{6} \lambda^{2}}{2} l_{3}(\kappa \mu)+\frac{3 \mu \lambda^{2}}{8 \kappa} l_{3}(\kappa \mu)+\frac{3 \omega \lambda^{2}}{16 \kappa} r_{31}+18 \kappa \lambda \lambda_{6}^{2} l_{3}(\kappa \lambda)-\frac{\lambda \lambda_{6}}{4}\left[6(N-2) l_{2}(0)\right. \\
& \left.+45 l_{2}(\kappa \lambda)+6 l_{2}(\kappa \mu)+3 r_{20}\right]+12 \kappa \lambda \mu_{6}^{2} l_{3}(\kappa \mu)+\frac{3 \lambda \mu^{2}}{4 \kappa} l_{3}(\kappa \mu)-6 \lambda \mu_{6} l_{2}(\kappa \mu)+6 \lambda \mu_{6} \mu l_{3}(\kappa \mu)+\frac{3 \lambda \omega^{2} r_{32}}{8 \kappa}+\frac{\omega^{3} r_{33}}{4 \kappa} \\
& \partial_{t} \mu_{6}=(2 d-4+3 \eta) \mu_{6}+\frac{\lambda^{3}}{16}\left[3 u_{21}(\kappa \lambda, \kappa \mu)+u_{21}(\kappa \mu, \kappa \lambda)\right]+\frac{1}{8}\left(-\frac{u_{11}(\kappa \lambda, \kappa \mu)}{\kappa}+\frac{3 l_{2}(\kappa \lambda)}{2 \kappa}-\frac{l_{2}(\kappa \mu)}{2 \kappa}+4 \kappa u_{21}(\kappa \lambda, \kappa \mu) \lambda_{6}\right. \\
& \left.+24 \kappa u_{21}(\kappa \lambda, \kappa \mu) \mu_{6}+12 \kappa u_{21}(\kappa \mu, \kappa \lambda) \mu_{6}+6 u_{21}(\kappa \lambda, \kappa \mu) \mu+3 u_{21}(\kappa \mu, \kappa \lambda) \mu\right) \lambda^{2}+\frac{1}{8}\left(96 u_{21}(\kappa \lambda, \kappa \mu) \mu_{6}^{2} \kappa^{2}\right. \\
& +96 u_{21}(\kappa \mu, \kappa \lambda) \mu_{6}^{2} \kappa^{2}+64 u_{21}(\kappa \lambda, \kappa \mu) \lambda_{6} \mu_{6} \kappa^{2}+16 u_{21}(\kappa \lambda, \kappa \mu) \lambda_{6} \mu \kappa+48 u_{21}(\kappa \lambda, \kappa \mu) \mu_{6} \mu \kappa+48 u_{21}(\kappa \mu, \kappa \lambda) \mu_{6} \mu \kappa \\
& +6 u_{21}(\kappa \lambda, \kappa \mu) \mu^{2}+6 u_{21}(\kappa \mu, \kappa \lambda) \mu^{2}+l_{3}(0) \mu^{2}+4 l_{2}(\kappa \lambda) \lambda_{6}-4 l_{2}(0) \mu_{6}-30 l_{2}(\kappa \lambda) \mu_{6}-24 l_{2}(\kappa \mu) \mu_{6}-8 u_{11}(\kappa \lambda, \kappa \mu)\left(\lambda_{6}\right. \\
& \left.\left.+4 \mu_{6}\right)-2 \mu_{6} r_{20}+2 \omega^{2} r_{212}(\kappa \mu)+\frac{2 u_{11}(\kappa \lambda, \kappa \mu) \mu}{\kappa}-\frac{3 l_{2}(\kappa \lambda) \mu}{2 \kappa}-\frac{l_{201}(\kappa \mu) \omega}{2 \kappa}-\frac{\mu r_{20}}{2 \kappa}+\frac{\omega r_{21}}{2 \kappa}\right) \lambda \\
& +\frac{1}{8}\left(256 \mu_{6}^{2}\left[u_{21}(\kappa \lambda, \kappa \mu) \lambda_{6}+u_{21}(\kappa \mu, \kappa \lambda) \mu_{6}\right] \kappa^{3}+192 u_{21}(\kappa \mu, \kappa \lambda) \mu_{6}^{2} \mu \kappa^{2}+128 u_{21}(\kappa \lambda, \kappa \mu) \lambda_{6} \mu_{6} \mu \kappa^{2}\right. \\
& +16 u_{21}(\kappa \lambda, \kappa \mu) \lambda_{6} \mu^{2} \kappa+48 u_{21}(\kappa \mu, \kappa \lambda) \mu_{6} \mu^{2} \kappa-16 \mu_{6}\left[4 u_{11}(\kappa \lambda, \kappa \mu) \lambda_{6}+5 l_{2}(\kappa \lambda) \lambda_{6}+16 u_{11}(\kappa \lambda, \kappa \mu) \mu_{6}\right. \\
& \left.+10 l_{2}(\kappa \mu) \mu_{6}\right] \kappa+4 u_{21}(\kappa \mu, \kappa \lambda) \mu^{3}-4 l_{2}(\kappa \lambda) \lambda_{6} \mu-8 l_{2}(0) \mu_{6} \mu-32 l_{2}(\kappa \mu) \mu_{6} \mu-16 u_{11}(\kappa \lambda, \kappa \mu)\left(\lambda_{6}+4 \mu_{6}\right) \mu \\
& -4 l_{201}(\kappa \mu) \mu_{6} \omega-4 \mu_{6} \omega r_{21}+4 \omega^{3} r_{213}(\kappa \mu)-\frac{u_{11}(\kappa \lambda, \kappa \mu) \mu^{2}}{\kappa}+\frac{2 l_{2}(\kappa \mu) \mu^{2}}{\kappa}+\frac{4 l_{1}(\kappa \mu) \lambda_{6}}{\kappa}-\frac{4 l_{2}(\kappa \lambda) \lambda_{6}}{\kappa}-\frac{44 l_{1}(\kappa \mu) \mu_{6}}{\kappa} \\
& \left.-\frac{l_{201}(\kappa \mu) \mu \omega}{\kappa}-\frac{\mu^{2} r_{110}(\kappa \mu)}{\kappa}+\frac{2 \mu \omega r_{111}(\kappa \mu)}{\kappa}-\frac{\mu \omega r_{21}}{\kappa}-\frac{\omega^{2} r_{112}(\kappa \mu)}{\kappa}+\frac{\omega^{2} r_{22}}{\kappa}\right)
\end{aligned}
$$


Here, $\eta=-\partial_{t} \ln Z$ is the anomalous dimension, and the threshold functions appeared in the above expressions are

$$
\begin{aligned}
& l_{m n s}(X)=\frac{v_{d}}{d} T^{\prime} \sum_{i} \frac{-2 m\left(\frac{\varepsilon_{i}^{2}}{c_{3}^{2}}+\frac{d}{d+2}\right)^{s}}{\left(\frac{\varepsilon_{i}^{2}}{c_{1}^{2}}+1+X\right)^{m+1}\left(\frac{\varepsilon_{i}^{2}}{c_{1}^{2}}+1\right)^{n}}, \\
& r_{m n}=v_{d} \int_{0}^{1} d y y^{d-1} T^{\prime} \sum_{i} \frac{-2 m\left(\frac{\varepsilon_{i}^{2}}{c_{3}^{2}}+y^{2}\right)^{n}}{\left[\frac{\varepsilon_{i}^{2}}{c_{1}^{2}}+1+\omega \kappa\left(\frac{\varepsilon_{i}^{2}}{c_{3}^{2}}+y^{2}\right)\right]^{m+1}}, \\
& u_{m n}(X, Y)=\frac{v_{d}}{d} T^{\prime} \sum_{i} \frac{-2\left[(m+n)\left(\frac{\varepsilon_{i}^{2}}{c_{1}^{2}}+1\right)+m Y+n X\right]}{\left(\frac{\varepsilon_{i}^{2}}{c_{1}^{2}}+1+X\right)^{m+1}\left(\frac{\varepsilon_{i}^{2}}{c_{1}^{2}}+1+Y\right)^{n+1}}, \\
& w_{m n s}(X)=v_{d} \int_{0}^{1} d y y^{d-1} T^{\prime} \sum_{i} \frac{-2\left(\frac{\varepsilon_{i}^{2}}{c_{3}^{2}}+y^{2}\right)^{s}}{\left[\frac{\varepsilon_{i}^{2}}{c_{1}^{2}}+1+\omega \kappa\left(\frac{\varepsilon_{i}^{2}}{c_{3}^{2}}+y^{2}\right)\right]^{m}\left(\frac{\varepsilon_{i}^{2}}{c_{1}^{2}}+1+X\right)^{n}}, \\
& h_{m n s}(X)=\frac{v_{d}}{d} T^{\prime} \sum_{i} \frac{\left(\frac{\varepsilon_{i}^{2}}{c_{3}^{2}}+y^{2}\right)^{s}}{\left[\frac{\varepsilon_{i}^{2}}{c_{1}^{2}}+1+\omega \kappa\left(\frac{\varepsilon_{i}^{2}}{c_{3}^{2}}+y^{2}\right)\right]^{m}\left(\frac{\varepsilon_{i}^{2}}{c_{1}^{2}}+1+X\right)^{n}}, \\
& r_{m n s}(X)=v_{d} \int_{0}^{1} d y y^{d-1} T^{\prime} \sum_{i} \frac{-2\left[(m+n)\left(\frac{\varepsilon_{i}^{2}}{c_{1}^{2}}+1\right)+m X+n \omega \kappa\left(\frac{\varepsilon_{i}^{2}}{c_{3}^{2}}+y^{2}\right)\right]}{\left[\frac{\varepsilon_{i}^{2}}{c_{1}^{2}}+1+\omega \kappa\left(\frac{\varepsilon_{i}^{2}}{c_{3}^{2}}+y^{2}\right)\right]^{m+1}\left(\frac{\varepsilon_{i}^{2}}{c_{1}^{2}}+1+X\right)^{n+1}} \Xi^{s},
\end{aligned}
$$

with $v_{d}=d \pi^{d / 2} /\left[(2 \pi)^{d} \Gamma(d / 2+1)\right], \varepsilon_{n}=2 \pi n T^{\prime}$, and $\Xi=\varepsilon_{i}^{2} / c_{3}^{2}+y^{2} . r_{m n s}(X)$ is given by (A15) with $\Xi$ replaced by $y^{2} . r_{m n s}(X)$ is given by (A15) with $\Xi$ replaced by $\varepsilon_{i}^{2} / c_{3}^{2}$. Also, $l_{m}(X) \equiv l_{m 00}(X)$. The renormalized temperature $T^{\prime}$ obeys the RG equation $\partial_{t} T^{\prime}=-T^{\prime}$. In the absence of the six-body terms, and in the limit $T \rightarrow 0$, the above RG equations agree with those obtained by Delamotte et al. for the classical model truncated up to the fourth order terms ${ }^{46}$ The RG equations are nonperturbative in the sense that the beta functions [the right-hand sides of Eqs. (A1)-(A9)] do not explode even in the strong-coupling limit $\lambda$ $\rightarrow \infty, \mu \rightarrow \infty$. The one-loop results of the nonlinear sigma model are reproduced in this limit.

${ }^{1}$ A. P. Ramirez, Annu. Rev. Mater. Sci. 24, 453 (1994).

${ }^{2}$ J. Villain, Phys. Chem. Solids 11, 303 (1959).

${ }^{3}$ A. Yoshimori, J. Phys. Soc. Jpn. 14, 807 (1959).

${ }^{4}$ T. A. Kaplan, Phys. Rev. 116, 888 (1959).

${ }^{5}$ G. Toulouse, Commun. Phys. (London) 2, 115 (1977).

${ }^{6}$ J. Villain, J. Phys. C 10, 1717 (1977).

${ }^{7}$ P. W. Anderson, Mater. Res. Bull. 8, 153 (1973); P. Fazekas and

P. W. Anderson, Philos. Mag. 30, 432 (1974).

${ }^{8}$ H. Kawamura and S. Miyashita, J. Phys. Soc. Jpn. 53, 4138 (1984).
${ }^{9}$ H. Kawamura, Phys. Rev. B 38, 4916 (1988).

${ }^{10}$ H. Kawamura, J. Phys. Soc. Jpn. 54, 3220 (1985); 56, 474 (1987).

${ }^{11}$ H. Kawamura, J. Phys. Soc. Jpn. 60, 1839 (1991).

${ }^{12}$ B. Bernu, C. Lhuillier, and L. Pierre, Phys. Rev. Lett. 69, 2590 (1992).

${ }^{13}$ P. Azaria, B. Delamotte, and D. Mouhanna, Phys. Rev. Lett. 70, 2483 (1993).

${ }^{14}$ T. Bhattacharya, A. Billoire, R. Lacaze, and Th. Jolicoeur, J. Phys. (Paris), Colloq. 4, 181 (1994). 
${ }^{15}$ A. Mailhot, M. L. Plumer, and A. Caillé, Phys. Rev. B 50, 6854 (1994).

${ }^{16}$ D. Loison and H. T. Diep, Phys. Rev. B 50, 16453 (1994).

${ }^{17}$ M. Wintel, H. U. Everts, and W. Apel, Phys. Rev. B 52, 13480 (1995).

${ }^{18}$ S. A. Antonenko, A. I. Sokolov, and K. B. Varnashev, Phys. Lett. A 208, 161 (1995).

${ }^{19}$ J. T. Chalker, P. C. W. Holdsworth, and E. F. Shender, Phys. Rev. Lett. 68, 855 (1992).

${ }^{20}$ S. Sachdev, Phys. Rev. B 45, 12377 (1992).

${ }^{21}$ I. Ritchey, P. Chandra, and P. Coleman, Phys. Rev. B 47, R15342 (1993).

${ }^{22}$ C. Zeng and V. Elser, Phys. Rev. B 42, 8436 (1990); 51, 8318 (1995).

${ }^{23}$ P. Lecheminant, B. Bernu, C. Lhuillier, L. Pierre, and P. Sindzingre, Phys. Rev. B 56, 2521 (1997).

${ }^{24}$ F. Mila, Phys. Rev. Lett. 81, 2356 (1998).

${ }^{25}$ B. Canals and C. Lacroix, Phys. Rev. Lett. 80, 2933 (1998).

${ }^{26}$ M. Isoda and S. Mori, J. Phys. Soc. Jpn. 67, 4022 (1998).

${ }^{27}$ R. Moessner and J. T. Chalker, Phys. Rev. Lett. 80, 2929 (1998).

${ }^{28}$ Y. Yamashita and K. Ueda, Phys. Rev. Lett. 85, 4960 (2000).

${ }^{29}$ A. Koga and N. Kawakami, Phys. Rev. B 63, 144432 (2001).

${ }^{30}$ H. Tsunetsugu, J. Phys. Soc. Jpn. 70, 640 (2001).

${ }^{31}$ S. Fujimoto, Phys. Rev. Lett. 89, 226402 (2002); Phys. Rev. B 67, 235102 (2003).

${ }^{32}$ S. Fujimoto, Phys. Rev. B 72, 024429 (2005).

${ }^{33}$ K. S. Raman, R. Moessner, and S. L. Sondhi, Phys. Rev. B 72, 064413 (2005).

${ }^{34}$ G. Misguich, C. Lhuillier, B. Bernu, and C. Waldtmann, Phys. Rev. Lett. 81, 1098 (1998); Phys. Rev. B 60, 1064 (1999).

${ }^{35}$ W. LiMing, G. Misguich, P. Sindzingre, and C. Lhuillier, Phys. Rev. B 62, 6372 (2000).

${ }^{36}$ Y. Shimizu, K. Miyagawa, K. Kanoda, M. Maesato, and G. Saito, Phys. Rev. Lett. 91, 107001 (2003).

${ }^{37}$ S. Nakatsuji, Y. Nambu, H. Tonomura, O. Sakai, S. Jonas, C. Broholm, H. Tsunetsugu, Y. Qiu, and Y. Maeno, Science 309, 1697 (2005).

${ }^{38}$ P. Azaria, B. Delamotte, and T. Jolicoeur, Phys. Rev. Lett. 64, 3175 (1990).

${ }^{39}$ A. V. Chubukov, T. Senthil, and S. Sachdev, Phys. Rev. Lett. 72, 2089 (1994).

${ }^{40}$ J. Alicea, O. I. Motrunich, M. Hermele, and M. P. A. Fisher, Phys. Rev. B 72, 064407 (2005).
${ }^{41}$ G. Zumbach, Phys. Rev. Lett. 71, 2421 (1993).

${ }^{42}$ G. Zumbach, Nucl. Phys. B 413, 771 (1994).

${ }^{43}$ D. Loison and K. D. Schotte, Eur. Phys. J. B 14, 125 (2000).

${ }^{44}$ M. Itakura, J. Phys. Soc. Jpn. 72, 74 (2003).

${ }^{45}$ M. Tissier, B. Delamotte, and D. Mouhanna, Phys. Rev. Lett. 84, 5208 (2000).

${ }^{46}$ B. Delamotte, D. Mouhanna, and M. Tissier, Phys. Rev. B 69, 134413 (2004).

${ }^{47}$ A. Pelissetto, P. Rossi, and E. Vicari, Phys. Rev. B 63, 140414(R) (2001).

${ }^{48}$ P. Calabrese, P. Parruccini, and A. I. Sokolov, Phys. Rev. B 68, 094415 (2003).

${ }^{49}$ P. Calabrese, P. Parruccini, A. Pelissetto, and E. Vicari, Phys. Rev. B 70, 174439 (2004).

${ }^{50}$ V. P. Plakhty, J. Kulda, D. Visser, E. V. Moskvin, and J. Wosnitza, Phys. Rev. Lett. 85, 3942 (2000).

${ }^{51}$ S. Chakravarty, B. I. Haperin, and D. R. Nelson, Phys. Rev. B 39, 2344 (1989).

${ }^{52}$ J. A. Hertz, Phys. Rev. B 14, 1165 (1976).

${ }^{53}$ A. P. Young, J. Phys. C 8, L309 (1975).

${ }^{54}$ T. Dombre and N. Read, Phys. Rev. B 39, 6797 (1989).

${ }^{55}$ P. Azaria, B. Delamotte, F. Delduc, and T. Jolicoeur, Nucl. Phys. B 408, 486 (1993).

${ }^{56}$ N. D. Mermin and H. Wagner, Phys. Rev. Lett. 17, 1133 (1966); S. Coleman, Commun. Math. Phys. 31, 259 (1973).

${ }^{57}$ C. Wetterich, Nucl. Phys. B 352, 529 (1991).

${ }^{58}$ N. Tetradis and C. Wetterich, Nucl. Phys. B 422, 541 (1994).

${ }^{59}$ J. Berges, N. Tetradis, and C. Wetterich, Phys. Rep. 363, 223 (2002).

${ }^{60}$ F. J. Wegner and A. Houghton, Phys. Rev. A 8, 401 (1973).

${ }^{61}$ C. Wetterich, Phys. Lett. B 301, 90 (1993).

${ }^{62}$ J. Polchinski, Nucl. Phys. B 231, 269 (1984).

${ }^{63}$ T. M. Morris, Int. J. Mod. Phys. A 14, 2411 (1994).

${ }^{64}$ B. W. Southern and A. P. Young, Phys. Rev. B 48, R13170 (1993).

${ }^{65}$ The role of the $Z_{2}$ vortex was, originally, discussed by Kawamura and Miyashita (Ref. 8) in connection with a possibility of KTlike phase transition in 2D HAF. As a matter of fact, KT-like low temperature critical phases caused by binding of $Z_{2}$ vortices do not exist, because in $2 \mathrm{D}$ systems the presence of continuous critical lines is allowed only in the case with $U(1)$ symmetry which is the class of the $c=1$ conformal field theory. 